\title{
The Relationship among Pulse Wave Velocity, Ankle-Brachial Pressure Index and Heart Rate Variability in Adult Males

\author{
Jeong-Hwan $\mathrm{Ahn}^{1}$, Mihee Kong ${ }^{1,2, *}$
}

Department of Family Medicine, ${ }^{1}$ Jeju National University Hospital, ${ }^{2}$ Jeju National University School of Medicine, Jeju, Korea

Background: Pulse wave velocity (PWV) and ankle-brachial pressure index (ABI) are non-invasive tools to measure atherosclerosis and arterial stiffness. Heart rate variability (HRV) has proven to be a non-invasive powerful tool in the investigation of the autonomic cardiovascular control. Therefore, the purpose of this study was to determine the relationship among PWV, ABI, and HRV parameters in adult males.

Methods: The study was carried out with 117 males who visited a health care center from April, 2009 to May, 2010. We conducted blood sampling (total cholesterol, triglyceride, high density lipoprotein, cholesterol, fasting glucose) and physical examination. We studied brachial-ankle PWV (baPWV) and ABI. We examined HRV parameters including standard deviation of NN interval (SDNN), low frequency (LF), high frequency (HF), LF/HF ratio. We analyzed the relationship among baPWV, ABI, and HRV parameters.

Results: SDNN had a significant negative correlation with age, systolic blood pressure and heart rate. LF and HF had a significant negative correlation with age, and age and heart rate, respectively. baPWV was significantly and positively associated with age, systolic and diastolic blood pressures, total cholesterol, fasting glucose and heart rate. ABI was negative correlated significantly with systolic and diastolic blood pressures and heart rate. After adjusting for all associated variables, baPWV was not correlated with HRV parameters, but there was a significant positive association between SDNN and ABI ( $\mathrm{r}=0.195, \mathrm{P}=0.014)$.

Conclusion: SDNN of HRV parameters had a significant positive correlation with ABI.

Keywords: Heart Rate Variability; Pulse Wave Velocity; Ankle-Brachial Pressure Index

\section{INTRODUCTION}

Received: September 23, 2011, Accepted: November 14, 2011

*Corresponding Author: Mihee Kong

Tel: 82-64-717-1830, Fax: 82-64-717-1583

E-mail: MHKong@paran.com

Korean Journal of Family Medicine

Copyright (c) 2011 The Korean Academy of Family Medicine

(a) This is an open-access article distributed under the terms of the Creative Commons Attribution Non-Commercial License (http://creativecommons.org/licenses/by-nc/3.0) which permits unrestricted noncommercial use, distribution, and reproduction in any medium, provided the original work is properly cited.
Arterial stiffness has been known to play a substantial role in the development of cardiovascular diseases through the process of atherosclerosis, and it can be measured non-invasively with pulse wave velocity (PWV) and ankle-brachial pressure index (ABI) in adults. $^{1-4)}$

PWV not only reflects risks of adverse cardiovascular events but also predicts their prognosis.-5) $\mathrm{ABI}$ works as a predictive parameter for peripheral diseases with low $\mathrm{ABI}$ indicating high risks of coronary heart diseases and cerebrovascular accidents. ${ }^{6)}$ 
Heart rate variability (HRV) quantifies variations in the interval between each heart beat and subsequently helps evaluate modulation of autonomic nervous system that consists of sympathetic and parasympathetic system. ${ }^{7)}$ It has been reported that heart rate variability either by inappropriate activation of sympathetic nervous system or lowered parasympathetic tone is related to increased risks of cardiovascular diseases. ${ }^{8,9)}$ Despite numerous previous efforts ${ }^{10,11)}$ to prove that PWV, ABI, and HRV may serve as useful markers for adverse cardiovascular events, there seems to be a lack of proper domestic investigations on their relationships. In this regard, we aimed to explore the correlations of HRV with PWV and AVI in male adults.

\section{METHODS}

\section{Subjects}

The participants were male adults aged at least 20 years old who visited Jeju University Hospital from April 2009 to May 2010 and had HRV, ABI, and PWV checked. Individuals excluded from the study were as follows: subjects with arrhythmia on electrocardiogram that might affect autonomic nervous function, subjects on treatment for previously diagnosed cardiovascular illnesses, hypertension, or diabetes, subjects with an average of $\mathrm{ABI}$ less than or equal to 0.9 suggesting presumable obstructive arteriosclerosis that could lead to decrease of PWV.

\section{Anthropometric Measurements}

Body mass index was calculated with body height and weight based on the formula of $\mathrm{kg} / \mathrm{m}^{2}$. Waist circumference was determined by measuring tape placed around abdomen at the level of umbilicus while standing relaxed. Blood pressure was measured with automatic blood pressure monitor, FT-700R (Jawon Medical Co., Seoul, Korea), after sitting for 5 minutes at rest.

\section{Blood Tests and Life Style}

Blood was taken after fasting for 12 hours to check fast blood sugar and lipid profile including total cholesterol, triglyceride, and high density lipoprotein cholesterol. Life style questionnaire asked details on smoking status, alcohol consumption, exercise habit, and degree of stress. As for smoking, it questioned whether you are a current or ex-smoker. Regarding alcohol use, it asked whether you have drunk alcoholic beverage more than or equal to one day per week for the past month. Questions about exercise were like if you have done exercise more than thirty minutes each time with the frequency of once or more on a regular basis for the last month. When it comes to the level of stress, it was determined by a Korean version of Brief Encounter Psychosocial Instrument (BEPSI). ${ }^{12)}$

\section{HRV}

For this exam, subjects were advised to abstain from smoking and drinking any beverages containing caffeine and were told to fast for more than 12 hours before the test. Participants were sitting in a chair with eyes open and directed toward the wall and not speaking in a soundproof room, where measurements were performed under appropriate lighting and pleasant temperature. HRV was measured by a sophisticated equipment, BFM-5000 Plus (Medi-Core Co., Seoul, Korea) taking 5 minutes for SDNN (standard deviation of all normal sinus R-R intervals over 24 hours) as time domain, low frequency ( $\mathrm{LH}, 0.04$ to $0.15 \mathrm{~Hz}$ ), high frequency (HF, 0.15 to $0.40 \mathrm{~Hz}$ ), and $\mathrm{LH} / \mathrm{HF}$ ratio as frequency domain.

\section{Brachial-Ankle PWV (baPWV) and ABI}

baPWV and $\mathrm{ABI}$ were measured using automated analyzer, VP-1000 (Colin Co., Komaki, Japan). Subjects lie on their back after taking 5 minutes of rest, and then had their both upper arms and ankles wrapped around by cuffs containing sensors which record pulse wave generated from brachial arteries and tibial arteries. With data obtained from the device, the time delay $(\Delta \mathrm{T})$ between brachial artery and tibial artery waveform was determined. The distance from heart to both ankles and upper arms, La and Lb, respectively were calculated automatically according to individual's height. Subsequently, baPWV was determined by the fomula: baPWV $=\mathrm{La}-\mathrm{Lb} / \Delta \mathrm{T}(\mathrm{cm} /$ s) where $\mathrm{La}$ - Lb stands for distance between $\mathrm{La}$ and $\mathrm{Lb}$, the average of baPWV on each side was defined as mean baPWV. The $\mathrm{ABI}$ was calculated by the ratio of systolic blood pressure in ankles to systolic pressure in arms. Likewise, mean ABI was derived by taking mean of both ratios. 


\section{Statistics}

For statistical analysis, we used the SPSS ver. 18.0 (SPSS Inc., Chicago, IL, USA) and implemented Pearson's correlation analysis to determine correlations among HRV, baPWV, ABI, certain lab values, and other basic variables. After adjusting for residual variables, subsequent relations between $\mathrm{HRV}$ and both baPWV and ABI were analyzed by partial correlation. P-value of less than 0.05 was considered statistically significant.

\section{RESULTS}

\section{Baseline Characteristics of Subjects}

A total of 177 participants with average ages of 43 were involved and their clinical baseline characteristics are listed in Table 1.

\section{Correlations among HRV, Mean baPWV, Mean ABI, and Other Variables (Table 2)}

SDNN shows negative correlations with age $(r=-0.206, \mathrm{P}$ $=0.006)$, systolic blood pressure $(\mathrm{r}=-0.148, \mathrm{P}=0.050)$, and heart rate $(\mathrm{r}=-0.161, \mathrm{P}=0.032)$. Likewise, $\mathrm{LF}$ and age are in an inverse correlation with each other $(\mathrm{r}=-0.201, \mathrm{P}=0.007)$. As for $\mathrm{HF}$, it reveals negative correlations with age $(\mathrm{r}=-0.238, \mathrm{P}=$ $0.001)$ and heart rate $(\mathrm{HR} ; \mathrm{r}=-0.212, \mathrm{P}=0.005)$ with statistical significance. baPWV turns out to be positively correlated with age $(\mathrm{r}=0.407, \mathrm{P}<0.001)$, systolic blood pressure $(\mathrm{r}=0.425, \mathrm{P}$ $<0.001)$, diastolic blood pressure $(\mathrm{r}=0.419, \mathrm{P}<0.001)$, total cholesterol $(\mathrm{r}=0.173, \mathrm{P}=0.021)$, fasting blood sugar $(\mathrm{r}=0.223$, $\mathrm{P}=0.003)$, and heart rate $(\mathrm{r}=0.384, \mathrm{P}<0.001)$ with significance in statistics. $A B I$ has significant negative correlation with systolic blood pressure $(\mathrm{r}=-0.194, \mathrm{P}=0.010)$, diastolic pressure $(\mathrm{r}$ $=-0.180, \mathrm{P}=0.017)$, and heart rate $(\mathrm{r}=-0.389, \mathrm{P}<0.001)$. However, BEPSI score shows no correlation with any of HRV, PWV, and ABI.

\section{Values of HRV, baPWV, and ABI According to Life Style}

No significant differences were found for HRV, baPWV, and $\mathrm{ABI}$ according to smoking or exercise status (data not shown). For the variable of alcohol consumption, a group of subjects (HF, $215.5 \pm 245.9$ ) who drinks one or more days per week over the
Table 1. Baseline characteristics of subjects $(n=177)$.

\begin{tabular}{|c|c|}
\hline Characteristics & Values \\
\hline \multicolumn{2}{|l|}{ Continuous variables, mean \pm SE (range) } \\
\hline \multicolumn{2}{|l|}{ Heart rate variability parameters } \\
\hline SDNN (msec) & $41.6 \pm 1.6(8.3-202.6)$ \\
\hline $\mathrm{LF}\left(\mathrm{msec}^{2}\right)$ & $394.2 \pm 36.1(1.3-4,630.7)$ \\
\hline $\mathrm{HF}\left(\mathrm{msec}^{2}\right)$ & $193.5 \pm 16.6(1.2-1,216.7)$ \\
\hline $\mathrm{LF} / \mathrm{HF}$ & $3.6 \pm 0.3(0.2-22.7)$ \\
\hline Mean baPWV (cm/s) & $1,342.5 \pm 14.9(985.5-2,065.5)$ \\
\hline Mean ABI & $114.3 \pm 0.6(92.0-133.5)$ \\
\hline \multicolumn{2}{|l|}{ Physiological variables } \\
\hline Age (y) & $43.0 \pm 0.7(20-78)$ \\
\hline BMI $\left(\mathrm{kg} / \mathrm{m}^{2}\right)$ & $25.5 \pm 0.2(18.1-35.1)$ \\
\hline Systolic BP (mm Hg) & $127.1 \pm 1.1(94-170)$ \\
\hline Diastolic BP (mm Hg) & $78.7 \pm 0.9(54-114)$ \\
\hline Heart rate (beat/min) & $65.0 \pm 0.8(43-107)$ \\
\hline Total cholesterol (mg/dL) & $199.1 \pm 2.9(107-420)$ \\
\hline HDL cholesterol (mg/dL) & $48.7 \pm 0.8(22-83)$ \\
\hline Triglyceride (mg/dL) & $144.2 \pm 7.5(34-842)$ \\
\hline Fasting glucose (mg/dL) & $90.4 \pm 1.8(66-294)$ \\
\hline \multicolumn{2}{|l|}{ Physiological variables, scores } \\
\hline BEPSI score $(n=167)$ & $1.74 \pm 0.05(1.0-5.0)$ \\
\hline \multicolumn{2}{|l|}{ Dichotomized variables, no (\%) } \\
\hline Smoking ( 1 , current; 0 , past or never) & $67(37.9) / 110(62.1)$ \\
\hline Alcohol (1, >1/wk; 0, <1/wk) & $129(72.9) / 48(27.1)$ \\
\hline Exercise (1, never; 0, >1/wk) & $108(61.0) / 69(39.0)$ \\
\hline
\end{tabular}

SDNN: standard deviation of all normal sinus R-R intervals over 24 hours, LF: low frequency, HF: high frequency, LF/HF: ratio of power in LF/HF, baPWV: brachial- ankle pulse wave velocity, ABI: anklebrachial pressure index, BMI: body mass index, BP: blood pressure, HDL: high-density lipoprotein, BEPSI: Brief Encounter Psychosocial Instrument.

past month shows significantly higher value of HF than the other group (HF, 134.4 \pm 115.6$)$ of people who consumes less than the former group $(\mathrm{P}=0.003$ by independent $\mathrm{t}$-test $)$.

\section{Correlations of HRV with baPWV and ABI (Table 3) \\ baPWV shows no correlation with any of SDNN, LF, HF, and} $\mathrm{LF} / \mathrm{HF}(\mathrm{P}>0.05)$. ABI has positive correlations with SDNN (r 
Table 2. Correlation among HRV parameters, mean baPWV, mean ABI, and other variables $(\mathrm{n}=177)$.

\begin{tabular}{|c|c|c|c|c|c|c|}
\hline \multirow{3}{*}{ Variables } & \multicolumn{4}{|c|}{ HRV parameters } & \multirow{2}{*}{ Mean baPWV } & \multirow{2}{*}{ Mean ABI } \\
\hline & SDNN & $\mathrm{LF}$ & $\mathrm{HF}$ & $\mathrm{LF} / \mathrm{HF}$ & & \\
\hline & $\mathrm{r}$ & $\mathrm{r}$ & $\mathrm{r}$ & $\mathrm{r}$ & $\mathrm{r}$ & $\mathrm{r}$ \\
\hline Age & $-0.206^{*}$ & $-0.201^{*}$ & $-0.238^{\dagger}$ & -0.108 & $0.407^{\dagger}$ & 0.127 \\
\hline BMI & -0.014 & 0.016 & -0.046 & -0.067 & -0.038 & -0.003 \\
\hline Systolic BP & $-0.148^{*}$ & -0.129 & -0.109 & -0.089 & $0.425^{\dagger}$ & $-0.194^{*}$ \\
\hline Diastolic BP & -0.139 & -0.109 & -0.094 & 0.014 & $0.419^{\dagger}$ & $-0.180^{*}$ \\
\hline Heart rate & $-0.161^{*}$ & -0.101 & $-0.212^{*}$ & 0.123 & $0.384^{\dagger}$ & $-0.389^{\dagger}$ \\
\hline Total cholesterol & 0.069 & -0.007 & 0.057 & 0.008 & $0.173^{*}$ & -0.133 \\
\hline HDL cholesterol & 0.020 & 0.072 & -0.021 & 0.064 & 0.034 & -0.022 \\
\hline Triglyceride & 0.023 & 0.052 & -0.020 & -0.016 & 0.139 & -0.042 \\
\hline Fasting glucose & -0.065 & -0.029 & -0.124 & 0.039 & $0.223^{*}$ & -0.003 \\
\hline BEPSI score & 0.038 & 0.054 & 0.020 & 0.083 & 0.038 & -0.126 \\
\hline
\end{tabular}

HRV: heart rate variability, baPWV: Brachial- ankle pulse wave velocity, ABI: Ankle-Brachial pressure index, SDNN: standard deviation of all normal sinus R-R intervals over 24 hours, LF: low frequency, HF: high frequency, LF/HF: ratio of power in LF/HF, r: correlation coefficient, BMI: body mass index, BP: blood pressure, HDL: high-density, BEPSI: Brief Encounter Psychosocial Instrument.

${ }^{*} \mathrm{P}<0.05 .{ }^{\dagger} \mathrm{P}<0.001$. P-value by Pearson's correlation analysis.

Table 3. Correlation between mean baPWV, mean ABI, and HRV parameters $(\mathrm{n}=177)$.

\begin{tabular}{|c|c|c|c|c|c|c|c|c|}
\hline & \multicolumn{8}{|c|}{ HRV parameters } \\
\hline & \multicolumn{2}{|c|}{ SDNN } & \multicolumn{2}{|c|}{ LF } & \multicolumn{2}{|c|}{$\mathrm{HF}$} & \multicolumn{2}{|c|}{$\mathrm{LF} / \mathrm{HF}$} \\
\hline & $\mathrm{r}^{*}$ & P-value ${ }^{\dagger}$ & $\mathrm{r}$ & P-value & $\mathrm{r}$ & P-value & $\mathrm{r}$ & P-value \\
\hline \multicolumn{9}{|c|}{ Mean baPWV } \\
\hline Model $1^{\ddagger}$ & -0.089 & 0.238 & -0.105 & 0.163 & -0.125 & 0.097 & -0.027 & 0.719 \\
\hline Model $2^{\S}$ & 0.036 & 0.658 & 0.008 & 0.922 & -0.002 & 0.981 & 0.019 & 0.816 \\
\hline \multicolumn{9}{|l|}{ Mean ABI } \\
\hline Model $1^{\ddagger}$ & 0.190 & 0.011 & 0.077 & 0.308 & 0.151 & 0.045 & -0.155 & 0.040 \\
\hline Model $2^{\S}$ & 0.195 & 0.014 & 0.056 & 0.484 & 0.137 & 0.087 & -0.130 & 0.105 \\
\hline
\end{tabular}

baPWV: Brachial- ankle pulse wave velocity, ABI: Ankle-Brachial pressure index, HRV: heart rate variability, SDNN: standard deviation of all normal sinus R-R intervals over 24 hours, LF: low frequency, HF: high frequency, LF/HF: ratio of power in LF/HF.

${ }^{*}$ r: correlation coefficient. ${ }^{\dagger}$ P-value by partial correlation analysis. ${ }^{\ddagger}$ Model 1: unadjusted. ${ }^{5}$ Model 2: adjusted for age, systolic blood pressure, diastolic blood pressure, heart rate, total cholesterol, fasting glucose, Brief Encounter Psychosocial Instrument (BEPSI) scores, smoking, alcohol, and exercise grades.

$=0.190, \mathrm{P}=0.011)$ and $\mathrm{HF}(\mathrm{r}=0.151, \mathrm{P}=0.045)$, while negative with LF/HF ( $\mathrm{r}=-0.155, \mathrm{P}=0.040)$. Notably, ABI retains its significant positive correlation with SDNN even after adjusting for other variables $(r=0.195, P=0.014)$.

\section{DISCUSSION}

The key finding of this study is that SDNN, a component of HRV, is positively correlated with ABI with statistical significance. SDNN, one of variables that reflect HRV in time 
domain measure, not only provides information on prognosis of cardiovascular diseases but also serves as a potent indicator of the controllability of ANS. ${ }^{7-9)}$ As HRV is determined by the net regulatory effect of both sympathetic and parasympathetic system on sinoatrial node, it is considered to be a reliable noninvasive indicator of the autonomic nervous function. ${ }^{7)}$ With increasing number of reports on relations between HRV and cardiovascular illnesses, many of them say it is possible to predict the likelihood of cardiovascular diseases by state of inappropriate augmentation of sympathetic function and decrease of parasympathetic tone. ${ }^{8,9)}$ Besides time domain measure, frequency domain analysis works as another method of HRV analysis where RR interval fluctuation calculated with the help of cutting edge digital devices and computer gives insight into differentiation between sympathetic and parasympathic system. LF reflects the effect of both sympathetic and parasympathetic tone (but better representative of sympathetic system). HF mirrors status of vasovagal function on SA node under the control of parasympathetic nerves. As for LF/HR ratio, it seems to represent the delicate balance between two systems. ${ }^{13-15)}$ Though HF and LF/HF ratio show no statistically significant correlation with $\mathrm{ABI}$ after adjusting for associated variables in the study.

In this study, systolic blood pressure and heart rate were negatively associated with SDNN and mean ABI. Reduced $\mathrm{HRV}$ is associated with an increased risk of cardiac events. ${ }^{16)}$ So, increased SDNN have protective effect for cardiac disease. Therefore, SDNN is significantly higher in metabolically healthy obese individuals than at risk subjects. ${ }^{17)}$

$\mathrm{ABI}$ is a simple and noninvasive screening tool for peripheral arterial disease (PAD). ${ }^{18)}$ When PAD is defined as having more than $50 \%$ of stenosis of peripheral artery determined by angiography, $\mathrm{ABI}$ is known to have $90 \%$ and $98 \%$ of sensitivity and specificity, respectively. ${ }^{19)}$ According to diagnostic criteria, $\mathrm{ABI}$ less than 0.9 refers to $\mathrm{PAD},{ }^{6,20)}$ and is reportedly associated with presence of coronary heart diseases and their progressions. ${ }^{21}$ In addition, it serves as a risk factor for complications of coronary heart diseases, ${ }^{6)}$ with greater than $1.4 \mathrm{ABI}$ being known to be related to the increased mortality rate. ${ }^{20)} \mathrm{ABI}$ was positively associated with SDNN, after adjusting for blood pressure, heart rate, and other variables on our study. HRV reflects the condition of autonomic regulation. So, our study said that HRV was associated with peripheral arterial disease. Really, one studyshowed that peripheral arterial disease altered the HRV in cardiovascular patients. $^{22)}$

Unlike this study where baPWV showed no relations with HRV, a research led by Nakao et al. ${ }^{10)}$ revealed the weak correlation of baPWV with the activation of sympathetic system in young adults.

Our study has following limitations. Since we recruited subjects who visited a local university hospital, the findings may not be generalized to whole Korean population. Additionally, we had a small number of male subjects. Furthermore, as a crosssectional study, it had limitation to reveal causal relations among variables. Despite all these limitations, present study sheds some light on relations between SDNN that provides useful information on effects of ANS or stability of cardiovascular system and $A B I$ that serves as a risk factor for cardiovascular diseases. Hopefully, further comprehensive studies with large scale will be performed in the near future.

\section{REFERENCES}

1. Hansen TW, Li Y, Staessen JA, Jeppesen J, Rasmussen $\mathrm{S}$, Wang JG, et al. Independent prognostic value of the ambulatory arterial stiffness index and aortic pulse wave velocity in a general population. J Hum Hypertens 2008;22:214-6.

2. Lehmann ED. Clinical value of aortic pulse-wave velocity measurement. Lancet 1999;354:528-9.

3. Laurent S, Boutouyrie P, Asmar R, Gautier I, Laloux B, Guize $\mathrm{L}$, et al. Aortic stiffness is an independent predictor of allcause and cardiovascular mortality in hypertensive patients. Hypertension 2001;37:1236-41.

4. van Popele NM, Grobbee DE, Bots ML, Asmar R, Topouchian J, Reneman RS, et al. Association between arterial stiffness and atherosclerosis: the Rotterdam Study. Stroke 2001;32:454-60.

5. Imanishi R, Seto S, Toda G, Yoshida M, Ohtsuru A, Koide $Y$, et al. High brachial-ankle pulse wave velocity is an independent predictor of the presence of coronary artery disease in men. Hypertens Res 2004;27:71-8.

6. Chuang SY, Chen CH, Cheng CM, Chou P. Combined use of brachial-ankle pulse wave velocity and ankle-brachial index 
for fast assessment of arteriosclerosis and atherosclerosis in a community. Int J Cardiol 2005;98:99-105.

7. Heart rate variability: standards of measurement, physiological interpretation and clinical use. Task Force of the European Society of Cardiology and the North American Society of Pacing and Electrophysiology. Circulation 1996;93:104365.

8. Curtis BM, O'Keefe JH Jr. Autonomic tone as a cardiovascular risk factor: the dangers of chronic fight or flight. Mayo Clin Proc 2002;77:45-54.

9. Gorman JM, Sloan RP. Heart rate variability in depressive and anxiety disorders. Am Heart J 2000;140(4 Suppl):77-83.

10. Nakao M, Nomura K, Karita K, Nishikitani M, Yano E. Relationship between brachial-ankle pulse wave velocity and heart rate variability in young Japanese men. Hypertens Res 2004;27:925-31.

11. Perkins GM, Owen A, Swaine IL, Wiles JD. Relationships between pulse wave velocity and heart rate variability in healthy men with a range of moderate-to-vigorous physical activity levels. Eur J Appl Physiol 2006;98:516-23.

12. Yim JH, Bae JM, Choi SS, Kim SW, Hwang HS, Huh BY. The validity of modified Korean-translated BEPSI (Brief Encounter Psychosocial Instrument) as instrument of stress measurement in outpatient clinic. J Korean Acad Fam Med 1996; 17:42-53.

13. Cacioppo JT, Berntson GG, Binkley PF, Quigley KS, Uchino BN, Fieldstone A. Autonomic cardiac control. II. Noninvasive indices and basal response as revealed by autonomic blockades. Psychophysiology 1994;31:586-98.

14. Malliani A, Pagani M, Lombardi F, Cerutti S. Cardiovascular neural regulation explored in the frequency domain. Circulation 1991;84:482-92.
15. Malliani A, Lombardi F, Pagani M. Power spectrum analysis of heart rate variability: a tool to explore neural regulatory mechanisms. Br Heart J 1994;71:1-2.

16. Nagai N, Moritani T. Effect of physical activity on autonomic nervous system function in lean and obese children. Int J Obes Relat Metab Disord 2004;28:27-33.

17. Robillard ME, Bellefeuille P, Comtois AS, Aubertin-Leheudre $\mathrm{M}$, Karelis $\mathrm{AD}$. The metabolically healthy but obese postmenopausal woman presents a favourable heart rate variability profile. Scand Cardiovasc J 2011;45:316-20.

18. Piecuch T, Jaworski R. Resting ankle-arm pressure index in vascular diseases of the lower extremities. Angiology 1989;40:181-5.

19. Criqui MH, Denenberg JO, Bird CE, Fronek A, Klauber MR, Langer RD. The correlation between symptoms and noninvasive test results in patients referred for peripheral arterial disease testing. Vasc Med 1996;1:65-71.

20. Resnick HE, Lindsay RS, McDermott MM, Devereux RB, Jones KL, Fabsitz RR, et al. Relationship of high and low ankle brachial index to all-cause and cardiovascular disease mortality: the Strong Heart Study. Circulation 2004;109:7339.

21. Papamichael CM, Lekakis JP, Stamatelopoulos KS, Papaioannou TG, Alevizaki MK, Cimponeriu AT, et al. Ankle-brachial index as a predictor of the extent of coronary atherosclerosis and cardiovascular events in patients with coronary artery disease. Am J Cardiol 2000;86:615-8.

22. Goernig M, Schroeder R, Roth T, Truebner S, Palutke I, Figulla HR, et al. Peripheral arterial disease alters heart rate variability in cardiovascular patients. Pacing Clin Electrophysiol 2008;31:858-62. 